\title{
Heroin Epidemic and Acute Kidney Injury: An Under- Recognized but Important Consequence of Opioid Overdose
}

\author{
Mohammad A. Hossain a, Hetavi Mahida a , Attiya Haroon ${ }^{\mathrm{a}}$, Eric J. Costanzo ${ }^{\mathrm{a}}$, \\ James Consentino ${ }^{\mathrm{a}}$, Loay Salman ${ }^{\mathrm{b}}$, Arif Asif ${ }^{\mathrm{a}, \mathrm{c}}$
}

\begin{abstract}
Heroin abuse and overdose are increasing at an alarming rate in the United States. Due to its relatively low cost and ease of availability, over a million people are abusing this agent. What is most disturbing is the fact that heroin-associated deaths have tripled over the past 5 years. While heroin has a major negative impact on the cardiopulmonary system, acute kidney injury (AKI) following heroin overdose is emerging as a major problem. AKI increases mortality and is a major cause of the development of chronic kidney disease and its antecedent long-term mortality. Timely diagnosis of AKI and its treatment reduces mortality. In this article, we present two cases (a 25-year-old man and a 22-year-old woman) of heroin-induced AKI. Both presented with altered mental status, respiratory depression and low blood pressure. AKI was diagnosed by the treating internist in a timely fashion and optimally treated in the 25-year-old man. In the 22-year-old woman who presented to the emergency department, AKI could not be recognized. She was discharged home after the management of overdose with resolution of pulmonary and neurological issues. Six days later, she returned to the emergency department with shortness of breath, volume overload, and severe acute tubular necrosis, required hemodialysis and left the hospital with serum creatinine of $2.5 \mathrm{mg} /$ $\mathrm{dL}$ (estimated glomerular filtration rate $(\mathrm{eGFR})=46 \mathrm{~mL} / \mathrm{min})$. At a 7-month follow-up, this patient continues to have eGFR of $45 \mathrm{~mL} / \mathrm{min}$ (stage III chronic kidney disease). This article presents the mechanism of heroin-induced AKI as well as its management strategy and calls for heightened awareness for early diagnosis and prompt treatment.
\end{abstract}

Keywords: Heroin; Acute kidney injury; Opioid overdose

\section{Introduction}

The United States is experiencing an epidemic of opioid use

Manuscript submitted August 23, 2017, accepted September 5, 2017

${ }^{a}$ Department of Medicine, Jersey Shore University Medical Center, Neptune, NJ, USA

bDepartment of Nephrology, Albany Medical College, Albany, NY, USA

${ }^{\mathrm{c} C}$ Corresponding Author: Arif Asif, Department of Medicine, Jersey Shore University Medical Center, Seton Hall-Hackensack-Meridian School of Medicine, 1945 Route 33, Neptune, NJ, USA. Email: arif.asif@hackensackmeridian.org

doi: https://doi.org/10.14740/jmc2898w with nearly 12.5 million people abusing opioids [1-4]. A major contributor to this problem is prescription opioid abuse [2, 4]. $\mathrm{CDC}$ has revealed that over the last 15 years, there has been a $200 \%$ increase in the rate of drug overdose deaths involving opioids, particularly heroin [5]. Recent data are emphasizing an alarming increase in heroin abuse $[4,5]$. While there are multiple factors responsible for the abuse, heroin's relatively low price and increased availability are major facilitators [3-5]. While most age groups are affected, heroin abuse is particularly prevalent in the younger population. In 2015 alone, 828,000 people used heroin and an additional 135,000 used heroin for the first time [4]. This is particularly disturbing, as heroin-associated deaths have dramatically increased, with rates tripling during the past 5 years in the younger population [4-7].

Cardiopulmonary demise is a major cause of death in heroin overdose; however, acute kidney injury (AKI) is emerging as a major contributor [5, 6, 8-11]. It has been documented that the development of AKI is associated with a fourfold increase in death and that its timely diagnosis and treatment improves mortality [12]. In this article, we present two cases of young adults of heroin overdose that developed AKI and present a management strategy for heroin-induced AKI. Our article also calls for heightened awareness of heroin-induced AKI, its early recognition and prompt treatment in order to reduce AKIassociated morbidity and mortality.

\section{Case Reports}

\section{Case 1}

A 22-year-old white female was brought to the emergency department after she was found to have stopped breathing and lost consciousness while at a party. She had a history of drug abuse (heroin). She received naloxone by emergency medical services (EMS) with improvement of her breathing. Upon arrival to the emergency department, she regained consciousness. Her past medical history was unremarkable except for heroin abuse that started 2 years ago following prescription of a narcotic analgesic for knee injury during a soccer game and subsequent right knee surgery. She also had history of one admission to a rehabilitation facility. Physical examination at the emergency department revealed a blood pressure of 99/78 $\mathrm{mm} \mathrm{Hg}$, pulse of 106 beats/min, a respiratory rate of 10 breaths/min and oxygen saturation $\left(\mathrm{SpO}_{2}\right)$ of $98 \%$ at room air. Pupillary examination demonstrated bilaterally constricted pupils. Chest and heart 
Table 1. Laboratory Parameters of Patients With Acute Kidney Injury After Heroine Abuse

\begin{tabular}{|c|c|c|c|c|c|}
\hline \multirow{2}{*}{ Laboratory parameter } & \multicolumn{2}{|l|}{ Patient 2} & \multicolumn{3}{|c|}{ Patient 1} \\
\hline & Day 1 & Day 2 & Day 1 & Day 6 & Month 7 \\
\hline Hemoglobin (g/dL) & 15 & 14.8 & 14.8 & 13.2 & 12.4 \\
\hline $\mathrm{WBC}\left(10^{3} / \mu \mathrm{L}\right)$ & 29.1 & 8.9 & 7.8 & 7.0 & 7.1 \\
\hline Platelet $\left(10^{3} / \mu \mathrm{L}\right)$ & 276 & 270 & 256 & 240 & 244 \\
\hline Calcium $(\mathrm{mEq} / \mathrm{L})$ & 8.9 & 8.8 & 9.2 & 9.4 & 9.3 \\
\hline Sodium $(\mathrm{mEq} / \mathrm{L})$ & 146 & 144 & 143 & 135 & 137 \\
\hline Potassium $(\mathrm{mEq} / \mathrm{L})$ & 4.3 & 4.4 & 4.2 & 5.2 & 4.7 \\
\hline Chloride (mEq/L) & 102 & 100 & 97 & 94 & 96 \\
\hline Lactic acid (mg/dL) & 3.4 & 1.4 & - & Normal & - \\
\hline Urine examination & $\begin{array}{l}\text { No evidence of hematuria, proteinuria, } \\
\text { glucosuria, tubular cells, or granular, } \\
\text { tubular, red cell or white cell casts. }\end{array}$ & - & $\begin{array}{l}\text { Numerous tubular } \\
\text { cells, granular } \\
\text { and tubular casts. }\end{array}$ & $\begin{array}{l}1+\text { proteinuria. No } \\
\text { evidence of hematuria } \\
\text { or glucosuria. }\end{array}$ & - \\
\hline
\end{tabular}

WBC: white blood cell; BUN: blood urea nitrogen; CPK: creatine phosphokinase; AST: aspartate aminotransferase; ALT: alanine aminotransferase; eGFR: estimated glomerular filtration rate $(\mathrm{mL} / \mathrm{min})$.

examination was normal. Her neurological examination was normal except for right leg weakness (4/5). She did not demonstrate peripheral edema. Initial laboratory analysis showed a normal leukocyte, hemoglobin and platelet count. Her serum creatinine was found to be $1.6 \mathrm{mg} / \mathrm{dL}$, with normal electrolytes (Table 1). Urine drug screen was positive for opioid and urinalysis revealed numerous tubular cells, granular and tubular cell casts. Arterial blood gas (ABG) and chest radiograph were normal. Because of the loss of consciousness and right lower extremity weakness, a CT scan of the head was obtained which did not reveal any intra-cranial bleed and the right leg weakness disappeared. Patient was discharged home with a referral to a rehab center. Six days after discharge, patient returned to the emergency department with bilateral lower extremity edema, shortness of breath and anuria. Physical examination showed three plus bilateral lower extremity edema and bilateral lower lobe rales with a blood pressure of 150/94 mm Hg. Laboratory data revealed serum creatinine of $5.8 \mathrm{mg} / \mathrm{dL}$, bicarbonate of 14 $\mathrm{mEq} / \mathrm{L}$ and serum potassium of $5.2 \mathrm{mEq} / \mathrm{L}$. Electrocardiogram (EKG) was normal without any peaked T waves. Nephrology consultation was obtained. Renal ultrasound revealed no hydronephrosis and normal size kidneys (9.3 and $9.4 \mathrm{~cm})$, normal echogenicity and cortical thickness. Foley catheter placed in the emergency department did not drain any urine and bladder scan did not demonstrate distended bladder. Hemodialysis therapy was instituted. After three dialysis sessions, patient volume status and blood pressure normalized. Subsequently, she started making urine and left the hospital (7 days later) off dialysis but with serum creatinine of $2.5 \mathrm{mg} / \mathrm{dL}$ (estimated glomerular filtration rate (eGFR) of $46 \mathrm{~mL} / \mathrm{min}$ ). At a follow-up of 7 months, patient continued with an eGFR of 45 and as such had stage III chronic kidney disease (CKD).

\section{Case 2}

A 25-year-old white male (chronic heroin abuser for 4 years) was brought to the hospital by EMS. He was found on the kitchen floor by his mother. He was unresponsive and found to have agonal respiration. Given his history, EMS administered naloxone. The patient regained consciousness with improvement of respiratory status. Past medical history was significant for heroin abuse and multiple admissions to a rehabilitation facility. Physical examination at the emergency department revealed a blood pressure of $88 / 60 \mathrm{~mm} \mathrm{Hg}$, pulse of 110 beats/min, a respiratory rate of 8 breaths/min and oxygen saturation $\left(\mathrm{SpO}_{2}\right)$ of $87 \%$ with pink frothy secretions at the mouth. Pupillary examination demonstrated bilaterally constricted pupils. Chest examination revealed decreased air entry on the right side with a few rales at the base of the lung. He had no fever, raised jugular venous pressure or peripheral edema. Initial laboratory analysis showed leukocytosis of $29,100 / \mu \mathrm{L}$, serum creatinine of 2.3 $\mathrm{mg} / \mathrm{dL}$, lactic acid of 3.4, and mildly elevated liver functions test and normal electrolytes (Table 1). Urine drug screen was positive for opioid. ABG analysis showed respiratory acidosis with $\mathrm{pH}$ of 7.19 , partial pressure of carbon dioxide $\left(\mathrm{PaCO}_{2}\right)$ at $47 \mathrm{~mm} \mathrm{Hg}$, partial pressure of oxygen $\left(\mathrm{PaO}_{2}\right)$ at $86 \mathrm{~mm} \mathrm{Hg}$ and $\mathrm{SpO}_{2}$ of $93 \%$. Chest radiograph showed diffuse infiltrates in the right lung (Fig. 1). He was given additional doses of naloxone and placed on positive pressure ventilation in the emer- 


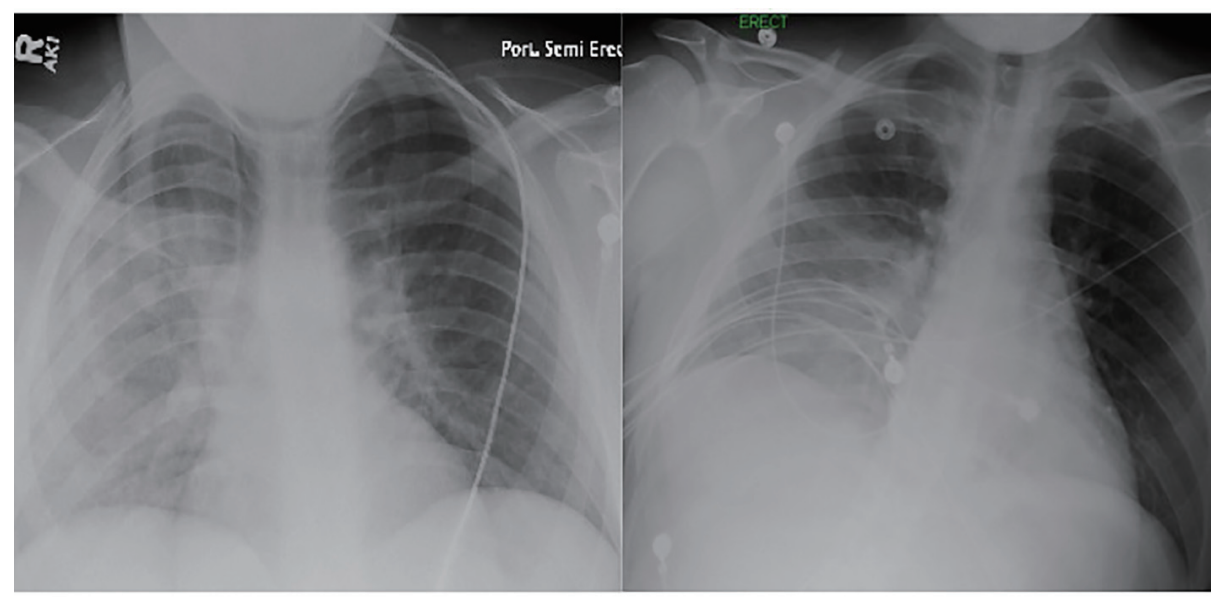

Figure 1. Chest X-ray performed at the time of admission revealing opacification and diffuse infiltrates of the right lung (left image). A repeat $X$-ray performed the next day demonstrated a significant clearance (right image).

gency department. The admitting internist diagnosed AKI due to dehydration and possible rhabdomyolysis. However, creatine phosphokinase (CPK) was found to be normal and urinalysis did not reveal any evidence in support of acute tubular necrosis. Suspecting AKI due to dehydration, the internist promptly replaced volume with normal saline administration $(1.5 \mathrm{~L}$ with careful attention to lung examination) with normalization of blood pressure to $132 / 88 \mathrm{~mm} \mathrm{Hg}$. The patient was also started on broad-spectrum antibiotic by the internist suspecting aspiration on the basis of leukocytosis and right-sided radiological findings on chest X-ray. On the second day of his hospitalization, oxygen saturation of $97 \%$ on room air, the patient's
ABGs were within normal ranges on room air. Renal function improved with a reduction of serum creatinine from 2.3 to 1.1 $\mathrm{mg} / \mathrm{dL}$ and resolution of leukocytosis. There was a dramatic improvement in air entry to the right lung and a repeat chest Xray showed complete resolution of right-sided infiltration (Fig. 2 ). Considering the non-infectious unilateral lung involvement associated with opioid abuse that clears without antimicrobial therapy, antibiotics were discontinued with continued improvement in patient condition. Echocardiogram showed normal left ventricular systolic function and mild liver dysfunction normalized. Patient was seen by drug rehab service during the hospital course and discharged home on day 4 with a referral to a reha-

Dehydration, hypotension and renal hypoperfusion

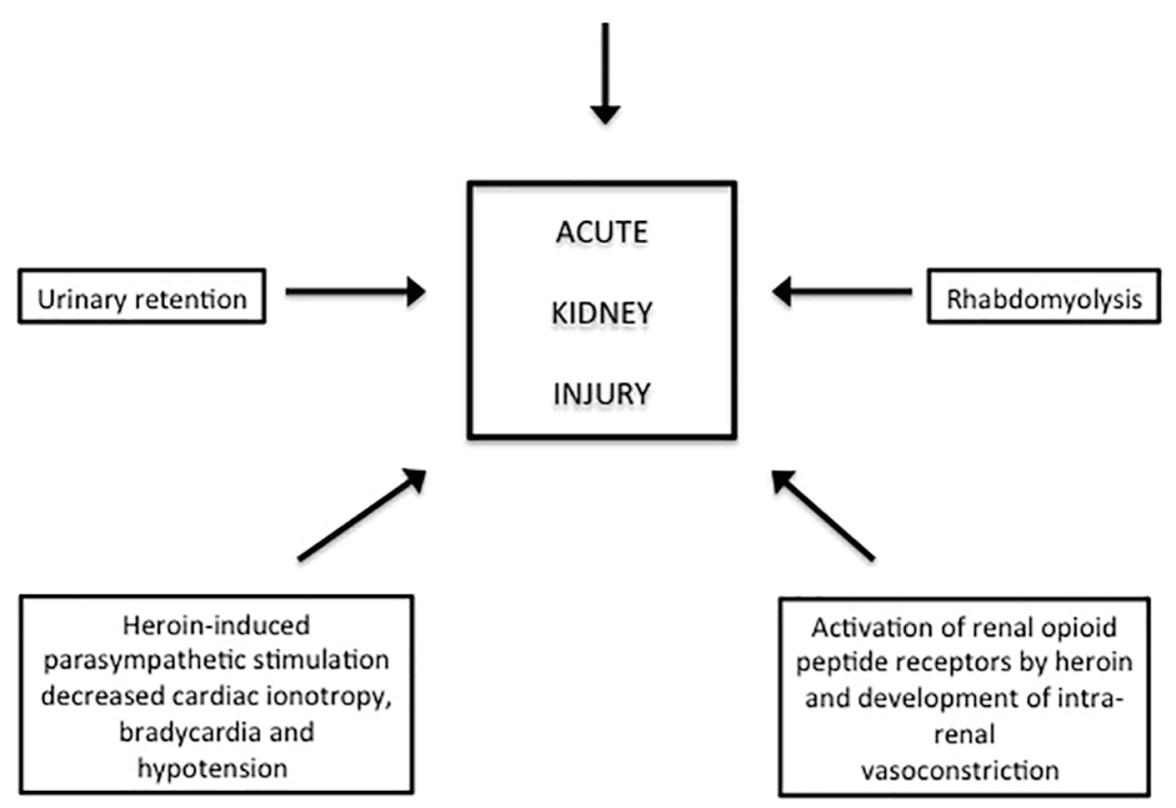

Figure 2. Mechanisms of heroin-induced acute kidney injury as elucidated by Mallappallil et al [8]. 
Patient with AKI in the context of heroin overdose

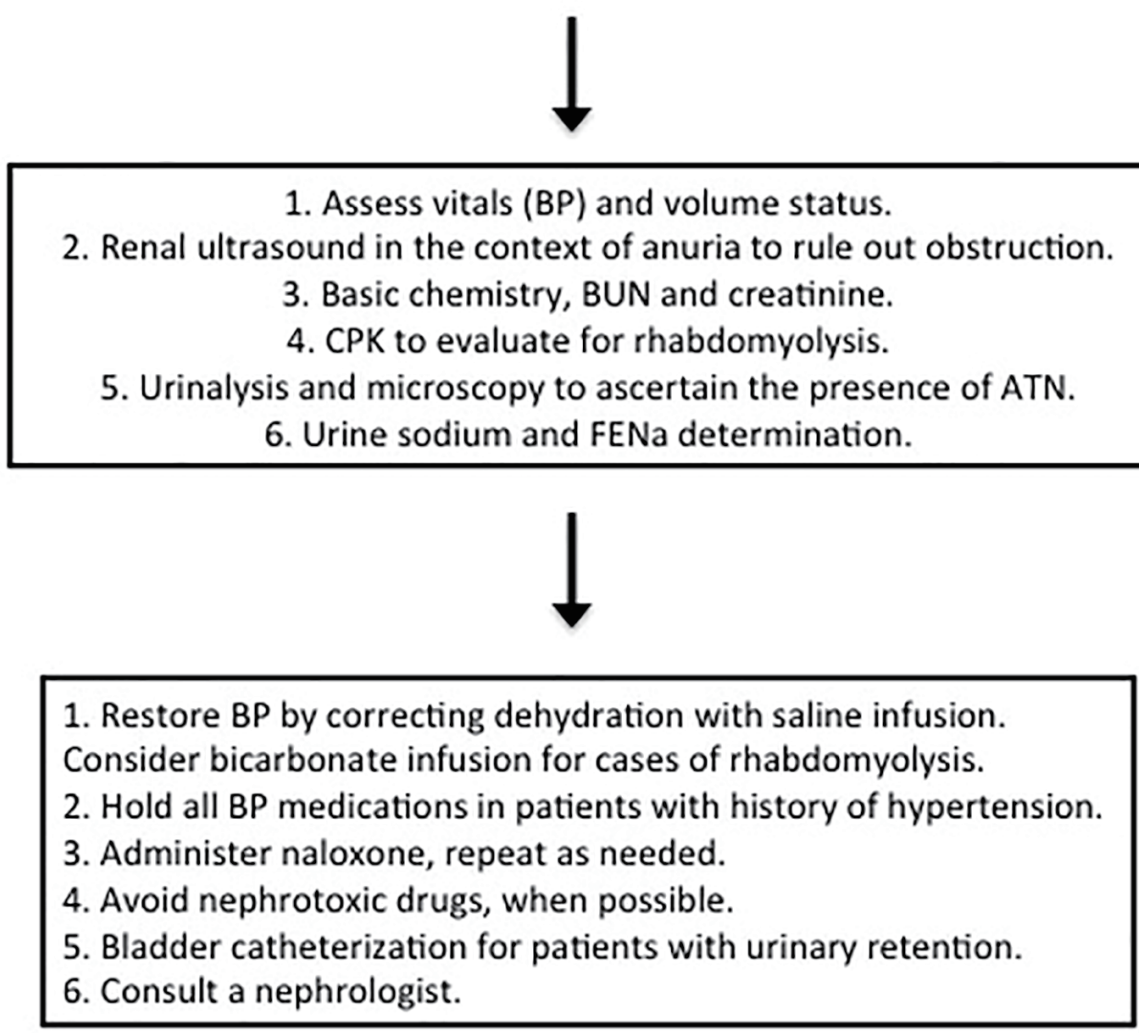

Figure 3. A simplified approach to heroin-induced AKI is presented. Replacement of intravascular volume, correction of blood pressure and restoration of renal blood flow are central to the management of AKI. Naloxone reduces intra-renal vasoconstriction and as such is a part of AKI management. Tubular cells, granular and tubular casts on urine microscopy are hallmarks of acute tubular necrosis (ATN). Muddy brown urine is also a feature of ATN. Myoglobinuria is seen with rhabdomyolysis resulting in red brown urine. Myoglobin is detected by urine dipstick as blood. However, there are only a few RBCs seen on urinalysis indicating that dipstick positivity is due to myoglobin and not blood itself. Fractional excretion of sodium (FENa) is a valuable tool in distinguishing between pre-renal and renal (ATN) cause of acute kidney injury. In pre-renal failure FENa is usually less than $1 \%$. On the other hand, values exceeding $2 \%$ indicated ATN. Values between $1 \%$ and $2 \%$ can be either pre-renal or renal AKI.

bilitation unit in a stable condition.

\section{Discussion}

Heroin (diacetylmorphinediacetylmorphine) is an extremely addictive opioid $[4,5]$. Its rapid and complete absorption results in its quick euphoric as well as toxic effects [1-5]. The two cases described here demonstrate the devastating effects of heroin. Both patients suffered from AKI. While the 25-yearold man was timely diagnosed and promptly treated for AKI with kidney function recovery, the renal damage was not initially appreciated in the 22-year-old woman. She left the hospital with advanced CKD. While acute heroin overdose can result in AKI, it is the chronic abuse that is more commonly associated with kidney injury [8]. This is due to the fact that with chronic abuse the drug and its metabolites are distributed and accumulated in multiple body compartments [8]. Both of our patients were chronic abusers of heroin for more than 2 years and hence more susceptible to renal damage.

In their elegant paper, Mallappallil et al have recently elucidated multiple mechanisms by virtue of which heroin can cause AKI [8] (Fig. 2). Opioids can lead to a decrease in fluid intake resulting in dehydration. Opioids also increase vagal activity as well as parasympathetic stimulation resulting in decreased cardiac ionotropy, bradycardia and hypotension [811]. Dehydration and hypotension act in concert to induce renal hypoperfusion and hence intra-renal vasoconstriction. Opioids also activate the so-called renal opioid peptide receptors and directly cause intra-renal vasoconstriction [8]. The intense intra-renal vasoconstriction can cause AKI. Both of our patients had hypotension and admitted to decreased fluid, as well as food intake. Rhabdomyolysis (particularly with immobility) and urinary retention (due to anti-cholinergic effects) can also be seen with opioids [8]. The 25-year-old man was found on the kitchen floor; however, the CPK was normal.

The timely diagnosis of AKI and its prompt management is critically important to reduce morbidity and mortality as- 
sociated with renal failure [12] (Fig. 3). In the 25-year-old man reported here, accurate diagnosis of AKI and its treatment with immediate saline replacement restored circulatory volume, improved blood pressure, improved renal perfusion, resolved intra-renal vasoconstriction and ameliorated ongoing renal injury. Naloxone administration also negates intra-renal vasoconstriction by reversing the effects of heroin on the opioid peptide receptors $[8,13]$. In contrast, the AKI encountered in the 22-year-old woman was not recognized during the initial admission. In fact, the urinalysis revealed clear evidence of acute tubular necrosis (ATN) as demonstrated by the presence of tubular cells, granular and tubular casts. Indeed, these urinary findings are virtually diagnostic of ATN $[14,15]$. This patient returned to the hospital with advanced kidney injury and needed dialysis therapy. The patient left the hospital with CKD stage III and will continue to require ongoing renal care to slow down the progression of CKD. CKD is also associated with a higher mortality than those without renal disease [16]. In this context, this patient will continue to carry a higher mortality profile. It is worth mentioning that heroin can also cause focal segmental glomerulonephritis [17]. However, this is commonly seen in blacks and presents with nephrotic range proteinuria. This was not the case in the patients reported here.

Interestingly enough the 25-year-old patient also had hypoxia and diffuse right lung pulmonary infiltrates. This complication is known as non-cardiogenic pulmonary edema (NCPE) and was first described by William Osler during an autopsy in 1880 [18]. NCPE is associated with opiate overdose, particularly with heroin and presents with significant hypoxia and diffuse pulmonary infiltrates within $24 \mathrm{~h}$ of overdose. The pulmonary infiltrates are not due to infection (pneumonia), can be bilateral or unilateral and resolve within $48 \mathrm{~h}$ [19]. Multiple recent reports have documented unilateral pulmonary infiltrate associated with a high leukocyte count following heroin overdose [19-22]. Both resolve spontaneously within $48 \mathrm{~h}$ and are not associated with infection [19-22]. In the 25-year-old male, after normal physical examination on day 2, the internist obtained a chest X-ray to document the disappearance of infiltrates. The white count also normalized, providing the support to discontinue antibiotics.

\section{Conclusion}

Heroin-associated AKI is an under-recognized and under-reported condition. While there are multiple mechanisms, dehydration resulting in hypotension and reduced renal flow is the predominant culprit for the development of AKI. Prompt recognition and careful hydration (with normal saline) and naloxone administration (negates intra-renal vasoconstriction) are readily available, cost effective and circumvent renal demise. Heightened awareness of AKI is needed to optimally manage patients presenting with heroin overdose.

\section{Grant}

This project was not supported by any grant or funding agen- cies.

\section{References}

1. Martins SS, Sampson L, Cerda M, Galea S. Worldwide prevalence and trends in unintentional drug overdose: a systematic review of the literature. Am J Public Health2015;105(11):e29-49

2. Hernandez SH, Nelson LS. Prescription drug abuse: insight into the epidemic. Clin Pharmacol Ther2010;88(3):307317

3. Manchikanti L, Singh A. Therapeutic opioids: A ten-year perspective on the complexities and complications of the escalating use, abuse, and nonmedical use of opioids. Pain Phys2008;11(2):63-88

4. Opioids: the prescription drugs and herion overdose epidemic. Accessed 2017. https://www.hhs.gov/opioids/index.htm

5. Increases in drug and opioid overdose deaths - United States, 2000-2014. Accessed 2017. https://www.cdc.gov/ $\mathrm{mmwr} / \mathrm{preview} / \mathrm{mmwrhtml} / \mathrm{mm} 6450 \mathrm{a} 3 . \mathrm{htm}$

6. Vital signs: demographic and substance use trends among heroin users - United States, 2002-2013. Accessed 2017. https://www.cdc.gov/mmwr/preview/mmwrhtml/mm6426a3. $\mathrm{htm}$

7. Cicero TJ, Ellis MS, Surratt HL, Kurtz SP. The changing face of heroin use in the United States: a retrospective analysis of the past 50 years. JAMA Psychiatry2014;71(7):821-826

8. Mallappallil M, Sabu J, Friedman EA, Salifu M. What do we know about opioids and the kidney? Int J Mol Sci2017;18(1):223

9. Feng G, Luo Q, Guo E, Yao Y, Yang F, Zhang B, Li L. Multiple organ dysfunction syndrome, an unusual complication of heroin intoxication: a case report and review of literature. Int J Clin Exp Pathol2015;8(9):11826-11830

10. Zhao A, Tan M, Maung A, Salifu M, Mallappallil M. Rhabdomyolysis and acute kidney injury requiring dialysis as a result of concomitant use of atypical neuroleptics and synthetic cannabinoids. Case Rep Nephrol2015;2015:235982

11. Melli G, Chaudhry V, Cornblath DR. Rhabdomyolysis: an evaluation of 475 hospitalized patients. Medicine (Baltimore)2005;84(6):377-385

12. Wang HE, Muntner P, Chertow GM, Warnock DG. Acute kidney injury and mortality in hospitalized patients. Am J Nephrol2012;35(4):349-355

13. Mutoh J, Ohsawa M, Hisa $\mathrm{H}$. Effect of naloxone on ischemic acute kidney injury in the mouse. Neuropharmacology2013;71:10-18

14. Perazella MA, Coca SG, Kanbay M, Brewster UC, Parikh CR. Diagnostic value of urine microscopy for differential diagnosis of acute kidney injury in hospitalized patients. Clin J Am Soc Nephrol2008;3(6):1615-1619

15. Perazella MA, Coca SG, Hall IE, Iyanam U, Koraishy M, Parikh CR. Urine microscopy is associated with severity and worsening of acute kidney injury in hospitalized patients. Clin J Am Soc Nephrol2010;5(3):402-408

16. https://www.niddk.nih.gov. National institute of diabetes, 
digestive and kidney disease-NIH. Accessed 2017

17. Jaffe JA, Kimmel PL. Chronic nephropathies of cocaine and heroin abuse: a critical review. Clin J Am Soc Nephrol2006;1(4):655-667

18. Sternbach G. William Osler: narcotic-induced pulmonary edema. J Emerg Med1983;1(2):165-167

19. Sporer KA, Dorn E. Heroin-related noncardiogenic pulmonary edema : a case series. Chest2001;120(5):1628-1632

20. Katz S, Aberman A, Frand UI, Stein IM, Fulop M. Heroin pulmonary edema. Evidence for increased pulmonary cap- illary permeability. Am Rev Respir Dis1972;106(3):472474

21. Radke JB, Owen KP, Sutter ME, Ford JB, Albertson TE. The effects of opioids on the lung. Clin Rev Allergy Immunol2014;46(1):54-64

22. Dettmeyer R, Schmidt P, Musshoff F, Dreisvogt C, Madea B. Pulmonary edema in fatal heroin overdose: immunohistological investigations with $\mathrm{IgE}$, collagen IV and laminin - no increase of defects of alveolar-capillary membranes. Forensic Sci Int2000;110(2):87-96 